\title{
Incidência de mosca-branca nas culturas de soja e milho e possibilidade de refúgios em área de Cerrado
}

\author{
Incidence of whitefly on soybean and corn crops and possibility of refuge in Cerrado area
}

Incidencia de mosca blanca en cultivos de soja y maíz y posibilidad de refugio en la zona del

Cerrado

Recebido: 03/03/2021 | Revisado: 10/03/2021 | Aceito: 16/03/2021 | Publicado: 22/03/2021

\author{
Graciene Pereira de Sousa \\ ORCID: https://orcid.org/0000-0001-6344-4889 \\ Universidade Federal do Piauí, Brasil \\ E-mail: gracienepereira1405@gmail.com \\ Regilene Bento Barbosa \\ ORCID: https://orcid.org/0000-0003-1723-6020 \\ Universidade Federal do Piauí, Brasil \\ E-mail: regilene.b.barbosa@gmail.com \\ Paulo Henrique Amaral Araújo de Sousa \\ ORCID: https://orcid.org/0000-0001-7194-9852 \\ Universidade Estadual do Oeste do Paraná \\ E-mail: paullo_ap1@hotmail.com \\ Jaqueline Zanon de Moura \\ ORCID: https://orcid.org/0000-0002-3582-9834 \\ Universidade Federal do Piauí, Brasil \\ E-mail: JAQUELINE.ZANON.M@ @otmail.com \\ Sinevaldo Gonçalves de Moura \\ ORCID: https://orcid.org/0000-0001-7595-902X \\ Universidade Federal do Piauí, Brasil \\ E-mail: sinevaldo.moura@yahoo.com.br \\ Paula Barbosa dos Santos \\ ORCID: https://orcid.org/0000-0003-3057-4761 \\ Universidade Federal do Piauí, Brasil \\ E-mail: paulabarbosa.1597@hotmail.com \\ Aluisio Costa Silva \\ ORCID: https://orcid.org/0000-0003-2611-9985 \\ Universidade Federal do Piaú, Brasil \\ E-mail: costtaaluisio@gmail.com
}

\begin{abstract}
Resumo
A mosca-branca (Hemiptera: Aleyrodidae) representa um grupo de insetos sugadores polífagos que compõe o complexo de pragas que atacam a soja, tendo como principal espécie praga Bemisia tabaci (Gennadius, 1889). Objetivou-se com esta pesquisa registrar a flutuação populacional de Aleyrodidae em áreas de cultivo de milho e soja nos municípios de Currais e Bom Jesus-PI, relacionar a ocorrência da praga com fatores climáticos e investigar se as áreas de Cerrado ao lado dos cultivos agrícolas podem servir como abrigo dessa praga na região. Foram definidos doze pontos de coleta com armadilhas adesivas amarelas. O intervalo médio de coleta foi de 45 dias, mantendo as armadilhas em campo por 24 horas. Estimaram-se os índices de diversidade, equitabilidade, dominância e frequência da ordem Hemiptera e da família Aleyrodidae na ordem Hemiptera. Para Aleyrodidae foram elaborados gráficos correlacionando a ocorrência à precipitação semanal acumulada anteriormente à coleta. A família Aleyrodidae é mais dominante e frequente em área de cultivo de soja quando comparada à área de cultivo de milho; a população de Aleyrodidae tende a diminuir com o aumento da precipitação; em áreas de cultivo de soja e milho contínuas à área de Cerrado a população de Aleyrodidae tende a se concentrar na mata de Cerrado; a manutenção de áreas de Cerrado próximas à áreas de cultivo de soja e milho pode favorecer o manejo integrado da família Aleyrodidae e diminuir os riscos de surtos populacionais nas áreas de cultivo comercial.
\end{abstract}

Palavras-chave: Aleyrodidae; Flutuação populacional; Armadilha adesiva amarela.

\begin{abstract}
The whitefly (Hemiptera: Aleyrodidae) represents a group of polyphagous sucking insects that make up the complex of pests that attack soybeans, having as main pest specie Bemisia tabaci (Gennadius, 1889). The objective of this research was to register the population fluctuation of Aleyrodidae in areas of corn and soybean cultivation in the municipalities of Currais and Bom Jesus-PI, to relate the occurrence of the pest with climatic factors and to investigate
\end{abstract}


whether the Cerrado areas next to agricultural crops can serve as shelter from this pest in the region. Twelve collection points with yellow adhesive traps were defined. The average collection interval was 45 days, keeping the traps in the field for 24 hours. The indexes of diversity, equitability, dominance and frequency of the order Hemiptera and the family Aleyrodidae in the order Hemiptera were estimated. For Aleyrodidae graphs were drawn up correlating the occurrence with the weekly precipitation accumulated prior to collection. The Aleyrodidae family is more dominant and frequent in soybean cultivation area when compared to the corn cultivation area; the population of Aleyrodidae tends to decrease with increasing precipitation; in areas of soy and corn cultivation continuous to the Cerrado area the population of Aleyrodidae tends to be concentrated in the Cerrado forest; the maintenance of Cerrado areas close to the soybean and corn cultivation areas can favor the integrated management of the family Aleyrodidae and decrease the risks of population outbreaks in the commercial cultivation areas.

Keywords: Aleyrodidae; Population fluctuation; Yellow sticky trap.

\section{Resumen}

La mosca blanca (Hemiptera: Aleyrodidae) representa un grupo de insectos chupadores polífagos que forman el complejo de plagas que atacan a la soja, siendo la principal especie Bemisia tabaci (Gennadius, 1889). El objetivo de esta investigación fue registrar la fluctuación poblacional de Aleyrodidae en áreas de cultivo de maíz y soja en los municipios de Currais y Bom Jesus-PI, relacionar la ocurrencia de la plaga con factores climáticos e investigar si las áreas del Cerrado próximas a Los cultivos agrícolas pueden servir de refugio para esta plaga en la región. Se definieron doce puntos de recogida con trampas adhesivas amarillas. El intervalo promedio de recolección fue de 45 días, manteniéndose las trampas en el campo durante 24 horas. Se estimaron los índices de diversidad, equidad, dominancia y frecuencia del orden Hemiptera y de la familia Aleyrodidae en el orden Hemiptera. Para Aleyrodidae, se elaboraron gráficos que correlacionan la ocurrencia con la precipitación semanal acumulada antes de la recolección. La familia Aleyrodidae es más dominante y frecuente en el área de cultivo de soja en comparación con el área de cultivo de maíz; la población de Aleyrodidae tiende a disminuir con el aumento de las precipitaciones; en áreas de cultivo de soja y maíz contiguas al área del Cerrado, la población de Aleyrodidae tiende a concentrarse en el bosque Cerrado; el mantenimiento de las áreas del Cerrado cercanas a las áreas de cultivo de soja y maíz puede favorecer el manejo integrado de la familia Aleyrodidae y reducir los riesgos de brotes poblacionales en las áreas de cultivo comercial.

Palabras clave: Aleyrodidae; Fluctuación de la población; Trampa pegajosa amarilla.

\section{Introdução}

O cultivo e a produtividade de grãos de soja (Glycine max (L.) Merr.) vêm crescendo a cada ano (Ferrazza et al., 2020), e possui grande importância no desenvolvimento econômico da região do Cerrado Piauiense. Entretanto, existem vários fatores que interferem negativamente na produção dos campos de soja e ocasionam grandes prejuízos. Além de fatores climáticos, insetos pragas como a mosca-branca Bemisia tabaci (Gennadius, 1889) (Hemiptera: Aleyrodidae) biótipo B representam importante causa de redução da produção da cultura da soja (Moraes et al., 2009).

Os danos provocados por B. tabaci sobre plantas de soja podem ser diretos ou indiretos e resultam na redução da produção ou até mesmo a morte das plantas, principalmente quando a praga se encontra em altas densidades populacionais (Suekane et al., 2013). As moscas-brancas apresentam metamorfose incompleta, passam pelas fases de ovo, ninfa e adultos e colonizam a parte inferior das folhas (Moscardi et al., 2012).

Bemisia tabaci biótipo B tornou-se rapidamente uma das pragas agrícolas mais importantes no Brasil, causando danos a diversas culturas e perdas econômicas estimadas em 714 milhões de dólares ao ano (Oliveira et al., 2013). Os prejuízos de $B$. tabaci na soja, geralmente, ocorrem devido a sucção da seiva, da excreção de um melado rico em açúcares que serve de substrato para o desenvolvimento do fungo fumagina (Capnodium spp., Capnodiaceae) que reduz a atividade fotossintética da planta, e pela transmissão de viroses (Lopez et al., 2008).

Além da soja, as moscas-brancas podem ser encontradas em mais de 600 espécies de plantas, incluindo ornamentais, daninhas, silvestres e outras culturas de importância econômica (Oliveira et al., 2001). Dessa forma, plantas daninhas remanescentes nas áreas de cultivo, hospedeiros alternativos presentes em áreas nativas ou cultivos comerciais de hospedeiros não preferenciais, como o milho podem permitir a sobrevivência de moscas-brancas em altas populações nas áreas de cultivo ou próximas delas no período de entressafra. A intensidade de ocorrência de moscas-brancas nas culturas depende dos sistemas 
de cultivos explorados na região, da ocorrência de hospedeiros alternativos durante o período da entressafra e das condições climáticas prevalecentes antes e durante o cultivo da soja (Ávila \& Grigolli, 2014).

Períodos secos e quentes favorecem o desenvolvimento e a dispersão da mosca-branca e são observados surtos na estação seca e reduções populacionais em estações de alta precipitação pluviométrica (Inoue-Nagata et al., 2016). Por tratar-se de um inseto vetor, o monitoramento precoce da ocorrência da praga é fundamental para a implementação de táticas de controle efetivas (Iost Filho, 2019). No caso das moscas-brancas, o monitoramento com armadilhas adesivas amarelas pode complementar o monitoramento direto, uma vez que esse tipo de armadilha atrai adultos de mosca-branca (Cáceres, 2004).

Com base nessa realidade, surgiu o interesse em estudar a ocorrência de mosca-branca em áreas de cultivo e áreas de vegetação nativa do Cerrado Piauiense e investigar se as áreas nativas são abrigos dessa praga na região. O registro da ocorrência e o conhecimento do comportamento populacional da família Aleyrodidae na região constitui informação chave para estabelecimentos de planos de manejo integrado de pragas, visando a redução da utilização de produtos nocivos ao homem e a natureza. Sendo assim, objetivou-se com esta pesquisa registrar o comportamento populacional da comunidade de mosca-branca relacionada às áreas de cultivo em Currais e Bom Jesus-PI, relacionar a ocorrência da praga com fatores climáticos e investigar se as áreas de Cerrado estão servindo como abrigo dessa praga na região.

\section{Metodologia}

$\mathrm{O}$ trabalho realizado seguiu a metodologia de pesquisa em campo de natureza quantitativa, segundo proposto por Pereira et al. (2018). O experimento foi conduzido em duas áreas, na Serra das Laranjeiras (Currais - Área 1 - 8 33'30.56" S,

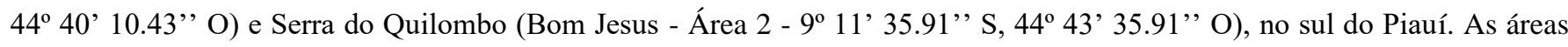
utilizadas foram de cultivo de milho (área 1) e soja (área 2) contínuas à área de vegetação nativa de Cerrado. As áreas naturais de Cerrado possuem graus semelhantes de preservação, fitofisionomia e tamanho de aproximadamente 500 hectares, próximas às áreas de cultivo. Em cada área foram definidos doze pontos de coleta dispostos na forma de um transecto em linha de 2900 metros. Os pontos de coleta foram colocados nas seguintes posições: quatro na área de cultivo $(150 \mathrm{~m}, 300 \mathrm{~m}, 600 \mathrm{~m}$ e $900 \mathrm{~m}$ da borda), e oito na área de Cerrado (1 na borda e 7 em direção ao centro da área, 150m, 300m, 600m, 900m, 1200m, 1500m e 2000m), o maior número de pontos dentro da mata tem o objetivo de avaliar a influência do raio (distância da borda) sobre a ocorrência do inseto (Figura 1).

Figura 1. Representação da localização das armadilhas adesiva amarela nas áreas de estudo, Currais e Bom Jesus-Piauí, 2015/2016.

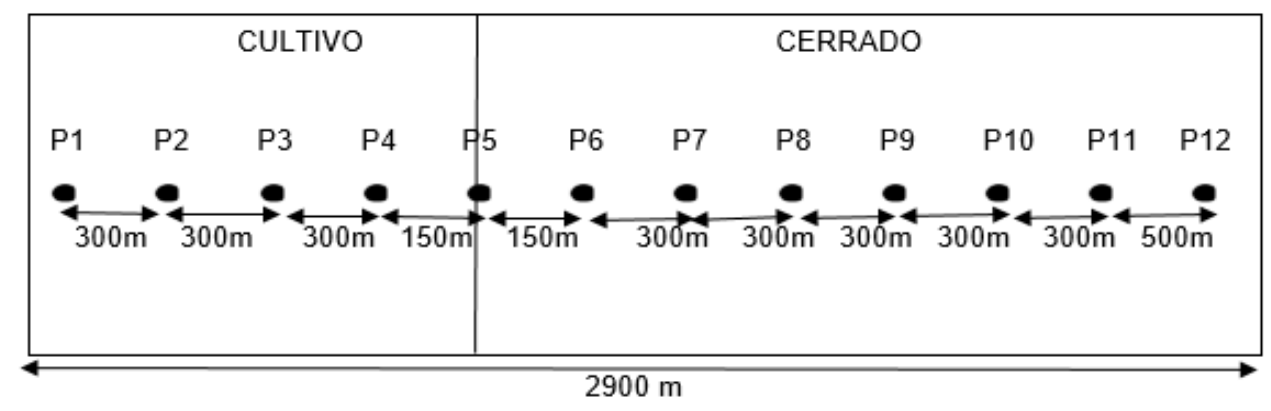

Fonte: Autores.

Em cada ponto foi instalado uma armadilha adesiva amarela "Trap" $(12 \times 21 \mathrm{~cm})$, sendo posicionada nas plantas da vegetação e em estacas na área de cultivo, na altura de $1 \mathrm{~m}$ a partir do solo. As armadilhas foram instaladas às 06:00 horas e 
após 24 horas foram retiradas e levadas ao laboratório, onde foi feita a triagem dos insetos. Foram realizadas sete coletas, no período de agosto de 2015 a julho de 2016, com intervalo médio de 45 dias.

As armadilhas foram conduzidas ao laboratório de Zoologia do Campus Professora Cinobelina Elvas da Universidade Federal do Piauí em gaiolas, onde ficavam fixadas com grampos em arames galvanizados, distanciadas a $0,3 \mathrm{~cm}$ uma das outras para evitar contato. Os insetos foram retirados das armadilhas com auxílio de pincel macio e pinça, utilizando água quente e detergente, triados por ordem e acondicionados em frascos com álcool 70\%. Posteriormente foram classificados por ordem de acordo com a chave dicotômica apresentada em Albertino (2012). Os pertencentes à ordem Hemiptera foram separados por morfoespécie e identificados em nível de família seguindo a chave dicotômica de Grazia et al. (2012). A confirmação da família Aleyrodidae foi realizada com auxílio do padrão das asas descrito por Csiro (2016).

Como há uma sazonalidade climática na região de estudo que se alterna entre períodos chuvosos e secos, os dados de precipitação foram coletados na fazenda, com auxílio de pluviômetro, utilizando-se para análise os dados cumulativos da semana antecessora da coleta.

Foi estimada a dominância da ordem Hemiptera em cada área, utilizando-se o índice de dominância de Berger e Parker (1970) e a frequência de ocorrência (FO) segundo Bodenheimer (1955), para a ordem coletada em cada área de estudo. Posteriormente estes índices foram calculados para a família Aleyrodidae dentro da ordem Hemiptera. Ainda para Aleyrodidae foram elaborados gráficos de flutuação populacional correlacionando a ocorrência da praga com dados de precipitação coletados na semana anterior às coletas.

\section{Resultados e Discussão}

Os índices de abundância e frequência da família Aleyrodidae, na ordem Hemiptera, foram maiores na área 2 (Tabela 1), comprovando a maior ocorrência desta família em cultivo de soja. A ordem Hemiptera é considerada dominante nas duas áreas com frequência primária. Já a família Aleyrodidae é acessória para ambas as áreas com frequência secundária na área 1 e primária na área 2.

Tabela 1. Dominância (D) e Frequência de Ocorrência (FO) da Ordem Hemiptera e da Família Aleyrodidae dentro da Ordem Hemiptera, em duas áreas formadas por segmento de cultivo e Cerrado, Currais e Bom Jesus-PI, 2015/2016.

\begin{tabular}{lcccc}
\hline & \multicolumn{2}{c}{ Área 1} & \multicolumn{2}{c}{ Área 2} \\
\hline Táxon & $\mathrm{D}$ & $\mathrm{FO}$ & $\mathrm{D}$ & FO \\
\hline Hemiptera & $26.5(303)$ & $71.4(12)$ & $15.2(305)$ & $100(12)$ \\
Aleyrodidae & $2.8(11)$ & $42.8(5)$ & $4.9(19)$ & $71.4(6)$ \\
\hline
\end{tabular}

D - Dominância (Berger, \& Parker, 1970); FO - Frequência de Ocorrência (Bodenheimer, 1955). Fonte: Autores.

B. tabaci é a principal espécie praga da família Aleyrodidae, esse inseto tornou-se praga de importância econômica para a cultura da soja devido às infestações crescentes e dificuldades de manejo (Vieira et al., 2012). A soja é uma de suas hospedeiras preferenciais (Inoue-Nagata et al., 2016).

Ainda segundo os mesmos autores, cucurbitaceas (abobrinha, melancia, melão e chuchu), solanáceas (tomate, berinjela, pimentão, tabaco, pimenta e jiló), brássicas (brócolos e repolho), leguminosas (feijão e feijão-vagem), algodão, mandioca, alface e quiabo, além de plantas ornamentais, daninhas e silvestres são hospedeiros da mosca-branca. Vieira et al. (2011), observaram que vários agricultores já tiveram grandes prejuízos devido à sua ocorrência, como redução da produção e 
aumento dos custos devido aos inseticidas usados para seu controle. Nesta relação, incluem os danos econômicos na soja, cuja área cultivada no País é 36,950 milhões de hectares (CONAB, 2020).

Durante os meses com maior precipitação a população de Aleyrodidae foi menor em ambas as áreas (Figura 2), ou seja, à medida que a precipitação aumenta a população (soma dos indivíduos coletados em todos os pontos) tende a diminuir, com índices de correlação $(\mathrm{R})$ entre número de indivíduos coletados e precipitação variando de mediano (A1) à forte (A2) corroborando com Leite et al. (2005).

Figura 2. Flutuação populacional de Aleyrodidae correlacionada à precipitação semanal acumulada (Área 1). Currais-PI, 2015/2016 (A). Flutuação populacional de Aleyrodidae correlacionada à precipitação semanal acumulada (Área 2). Bom JesusPI, 2015/2016 (B).

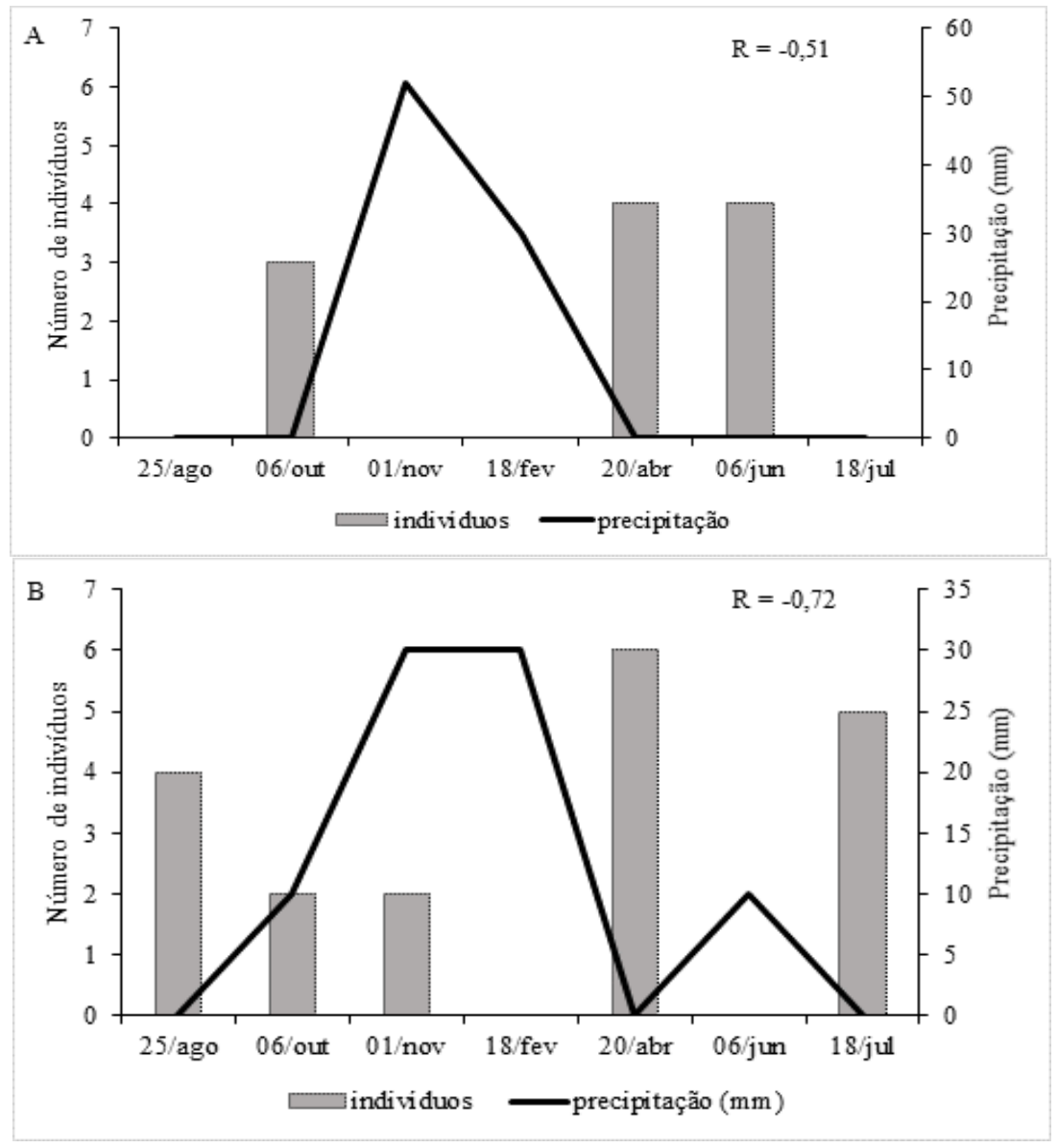

Fonte: Autores.

Observa-se na Figura 3 que, na área 1, nos pontos P1 e P2 que são os mais internos na área de lavoura (1050m e 750m da borda), coleta-se moscas-brancas apenas em abril de 2016, situação semelhante do P6 onde, no entanto, coletou-se um número maior de indivíduos. No ponto P5, localizado na divisa da área de cultivo e Cerrado coletou-se apenas em junho de 2016.

O pico de indivíduos coletados localiza-se no P9 no mês de outubro. Não ocorrendo registros nos demais pontos. Observa-se que os picos de coletas coincidem com os pontos localizados na área de Cerrado, demonstrando a utilização de plantas nativas como hospedeiros da família Aleyrodidae, principalmente após o fim das chuvas.

Com os resultados apresentados na Figura 3, na área 2, visualizamos situação semelhante. Nos pontos P1 e P2 (1050m e 750m internos na área de lavoura) não houve registro de mosca-branca, que começou a ser registrada a partir do ponto P3 
(450m internos na área de lavoura) até o ponto 6 (150m internos na área da mata). Nos pontos 7, 8 e 9 não foram registrados indivíduos, provavelmente por estarem próximos à uma estrada.

Figura 3. Flutuação populacional de Aleyrodidae por ponto de coleta em área formada por segmento de cultivo de milho e Cerrado (Área 1), Currais-PI. 2015/2016 (A). Flutuação populacional de Aleyrodidae por ponto de coleta em área formada por segmento de cultivo de soja e Cerrado (Área 2), Bom Jesus-PI. 2015/2016 (B).

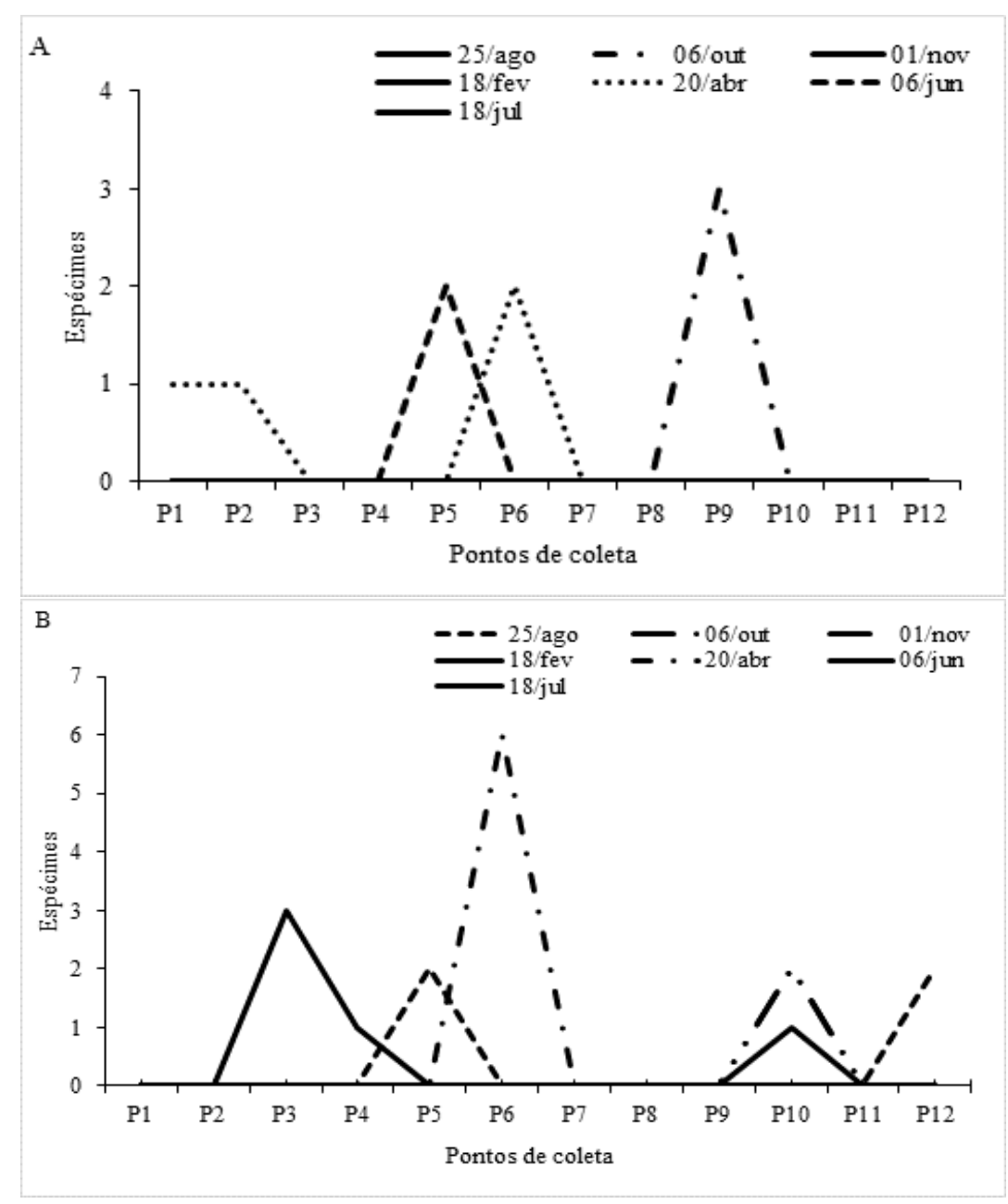

Fonte: Autores.

A flutuação populacional de Aleyrodidae, por ponto de coleta, no período de agosto de 2015 a julho de 2016 está representada Figura 4. Na área 1 o pico máximo da família ocorreu no ponto P9 (1050m internos na área da mata) durante o mês de outubro, ainda não havia cultura no campo. Na área 2 ocorreu no ponto P6 (150m internos na área da mata) durante o mês de abril, coincidindo com o final da cultura no campo. 
Figura 4. Ocorrência de Aleyrodidae por data nos doze pontos de coleta em área formada por segmento de cultivo de milho e Cerrado (Área 1), Currais-PI, 2015/2016 (A). Ocorrência de Aleyrodidae por data nos doze pontos de coleta em área formada por segmento de cultivo de soja e Cerrado (Área 2), Bom Jesus-PI, 2015/2016 (B).

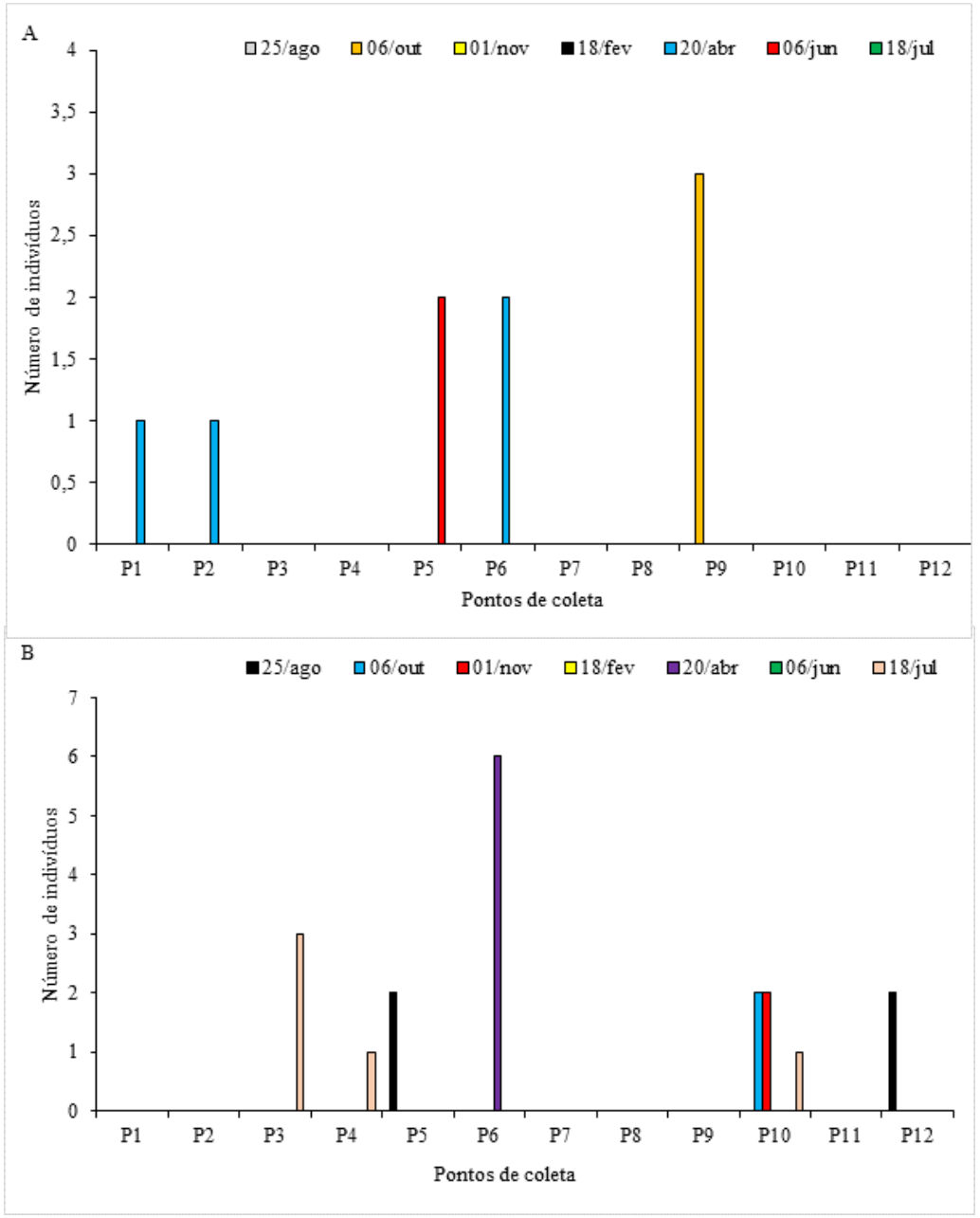

Fonte: Autores.

$\mathrm{Na}$ parte interna do Cerrado, nas duas áreas, ocorreram maiores registros de Aleyrodidae, com isso pode-se afirmar que a mosca-branca está utilizando as áreas de Cerrado como refúgio, mostrando que a preservação da mata nativa é ferramenta importante no manejo desta praga. De acordo com Oliveira e Wolski (2012) as áreas de Reserva Legal possuem um importante papel para a manutenção e preservação dos recursos ecológicos, ao manter uma área com mata o proprietário rural diminui a quantidade de pragas na plantação, garantindo abrigo e alimento para diversos animais que deixam de invadir as lavouras para se alimentar. Assim como, os corredores ecológicos que segundo Oliveira et al. (2015) são importantes instrumentos na conservação da biodiversidade em áreas nativas, principalmente em áreas florestadas e fragmentadas, exercendo a função de habitat e fornecendo condições ambientais para a reprodução e sobrevivência das espécies.

\section{Conclusão}

A família Aleyrodidae é mais dominante e frequente em área de cultivo de soja quando comparada à área de cultivo de milho.

A população de Aleyrodidae tende a diminuir com o aumento da precipitação. 
Em áreas de cultivo de soja e milho contínuas à área de Cerrado, a população de Aleyrodidae tende a se concentrar na mata de Cerrado.

A manutenção de áreas de Cerrado próximas às áreas de cultivo de soja e milho pode favorecer o manejo da família Aleyrodidae.

Considerando a importância da praga, estudos adicionais devem ser conduzidos com outras culturas e em outras áreas do Cerrado Piauiense afim de conhecer o comportamento populacional da família Aleyrodidae na região e assim auxiliar produtores locais no estabelecimento de planos de manejo integrado de pragas.

\section{Agradecimentos}

Ao CNPq pela concessão da bolsa de iniciação à pesquisa e à Universidade Federal do Piauí pela oportunidade de integrar o Programa Institucional de Bolsas de Iniciação Científica. Às pessoas que integram as fazendas São Carlos e União pela disponibilização das áreas de pesquisa e apoio recebido. À Doutoranda Cláudia Renata Madella-Auricchio pelo auxílio na instalação do experimento e coleta dos insetos. Aos colegas Kávio Karibe e Romário Bezerra pela colaboração na condução da pesquisa.

\section{Referências}

Albertino, R. J. (2012). Chaves para as ordens adulto. In: Albertino, R. J. (ed). Insetos do Brasil: Diversidade e taxonomia. Ribeirão Preto: Holos Editora, 810 p.

Ávila, C. J. \& Grigolli, J. F. J. (2014). Pragas da soja e seu controle. In: lourenção, A. L. F. et al. (eds). Tecnologia e produção: Soja 2013/2014. Curitiba: Midiograf, $247 \mathrm{p}$.

Berger, W. H. \& Parker, F. L. (1970). Diversity of planktonic foraminifera in deep-sea sediments. Science, 168, $1345-1347$.

Bodenheimer, F. S. (1955). "Précis d'ecalogie animale". Payot, 315 p.

Cáceres, S. (2004). Moscas blancas del complejo Bemisia tabaci en cultivos hortícolas de corrientes. Estrategia de manejo. En: Mosca blanca Bemisia tabaci. Jornada de Actualizacíon. Buenos Aires, pp. 9-13.

CONAB - Companhia Nacional de Abastecimento. (2020). Boletim Monitoramento Agrícola, Brasília, 9(9), 1-14.

CSIRO - Commonwealth Scientific and Industrial Research Organisation. Australian insect families. (2016). <http://anic.ento.csiro.au/insectfamilies> (Acesso em 12 jun 2016).

EMBRAPA. Tecnologias de produção de soja - Paraná - 2004. (2003). Embrapa Soja, 218 p.

Ferrazza, F. L. F., Jacoboski, D. T. K., Wyrepkowski, A., Rodrigues, L., Figueiró, A. G., \& Paraginski, R. T. (2020). Qualidade de sementes e parâmetros produtivos de sementes de soja submetidas a diferentes tratamentos de sementes antes da semeadura. Research, Society and Development, 9 (9), e47996232e47996232.

Grazia, J. Hemiptera. In: Rafael, A. J. et al. (eds.) (2012). Insetos do Brasil: Diversidade e taxonomia. 810 p.

Inoue-Nagata, A. K.; Lima, M. F. \& Gilbertson, R. L. (2016). A review of geminivirus (begomovirus) diseases in vegetables and other crops in Brazil: current status and approaches for management. Horticultura Brasileira. 34(1), 8-18.

Iost Filho, F. H. (2019). Sensoriamento remoto para monitoramento de mosca branca, Bemisia tabaci biótipo B (Hemiptera: Aleyrodidae) em soja. (Doctoral dissertation, Universidade de São Paulo).

Leite, G. L. D. (2005). Bemisia tabaci, Brevicoryne brassicae and Thrips tabaci abundance on Brassica oleracea var. acephala. Pesquisa Agropecuária Brasileira, Brasília, 40(3), 197-202.

Lopez, V. et al. (2008). Discovery learning about sustainable management of whitefly pests and whitefly-borne viruses. International Centre for Tropical Agriculture (CIAT), 1(1), 12-37.

Moraes J. C.; Ferreira R. S. \& Costa R. R. (2009). Indutores de resistência à mosca-branca Bemisia tabaci biótipo B (Genn., 1889) (Hemiptera: Aleyrodidae) em soja. Ciência e Agrotecnologia, 33(5), 1260-1264.

Moscardi, F. et al. (2012). Artrópodes que atacam as folhas da soja. In: Hoffman-Campo, C. B.; Corrêa-Ferreira, B. S. \& Moscardi, F. (Eds) Soja - manejo integrado de insetos e outros artrópodes praga. Brasília: Embrapa, 4, 211-334.

Oliveira, A. P. G. et al. (2015). Uso de geotecnologias para o estabelecimento de áreas para corredores de biodiversidade. Revista Árvore, 39(4), 595-602. 
Research, Society and Development, v. 10, n. 3, e48010313529, 2021

(CC BY 4.0) | ISSN 2525-3409 | DOI: http://dx.doi.org/10.33448/rsd-v10i3.13529

Oliveira, C. M. et al. (2013). Economic impact of exotic insect pests in Brazilian agriculture. Journal of Applied Entomology, 137(1-2), 1-15.

Oliveira, M. R. V.; Henneberry, T. J. \& Anderson, P. (2001). History current status, and collaborative researchprojects for Bemisia tabaci. Crop Protection, 20(9), 709-723.

Oliveira, T. \& Wolski, M. S. (2012). Importância da reserva legal para a preservação da biodiversidade. Vivências, 8(15), 40-52.

Pereira, A. S., Shitsuka, D. M., Parreira, F. J., \& Shitsuka, R. (2018). Metodologia da pesquisa científica. UFSM. https://repositorio.ufsm.br/bitstrea $\mathrm{m} /$ handle/1/ 15824/Lic_Computacao_MetodologiaPesquisa-Cientifica.pdf?sequence=1.

Suekane, R. et al. (2013). Danos da Mosca-Branca Bemisia Tabaci (Genn.) e distribuição vertical das ninfas em cultivares de soja em casa de vegetação. Arquivos do Instituto Biológico, 80(2), 151-158.

Vieira, S. S. et al. (2012). Resistance of soybean genotypes to Bemisia tabaci (Genn.) biotype B (Hemiptera: Aleyrodidae). Neotropical Entomology, 40(1), 117-122.

Vieira, S. S. et al. (2012). Efeitos dos inseticidas utilizados no controle de Bemisia tabaci (Gennadius) biótipo B e sua seletividade aos inimigos naturais na cultura da soja. Semina: Ciências Agrárias, 33(5), 1809-1818. 